\title{
Outcome of emergency one-stage resection and anastomosis procedure for patients with obstructed colorectal cancer
}

\author{
Yuko Sumise', Kazuo Yoshioka",2, Natsu Okitsu', Hitomi Kamo', Yusuke Arakawa', \\ Takeshi Yamaguchi', Yukari Harino', Yoshihiro Nakai', Akemi Yamanaka ${ }^{3}$, and \\ Seiki Tashiro ${ }^{1,4}$ \\ ${ }^{1}$ Department of Surgery, Taoka Hospital, Tokushima, Japan, ${ }^{2}$ Clinical Professor, Department of Digestive \\ and Transplantation surgery, the University of Tokushima Graduate School, ${ }^{3}$ Department of Anesthe- \\ siology and Emergency, Taoka Hospital, ${ }^{4}$ Professor Emeritus, the University of Tokushima
}

\begin{abstract}
Purposes : The purpose of this study was to verify the outcome of the emergency one stage resection and anastomosis procedure for patients with obstructed colorectal cancer. Methods : An emergency one stage resection and anastomosis procedure was performed for 40 patients with obstructive colorectal cancer. The outcome was verified and compared dividing into two groups. 17 patients under the age of 70 in (Group A), 23 patients 70 years and over in (Group B). Results : The operative mortality rate in both groups was $0 \%$. As a result, postoperative complications were not significantly different between the two groups. The overall survival rate after a 5 -year period in both groups was 41.8\%, regarding all patients and the survival curves for the two groups, was not significantly different. The 5 year survival rate in stage II or III showed no differences between the two groups. Conclusion : The one-stage resection and anastomosis of the large bowel could be applied safely to emergency patients, which in turn allows for excellent shortterm operative results in both groups mentioned. This particular procedure should be positively enforced, even in elderly patients in their 70's. J. Med. Invest. 60 : 249-255, August, 2013
\end{abstract}

Keywords : obstructed colorectal cancer, emergency one-stage resection and anastomosis, intraoperative intestinal decompression, elderly patients with aged 70 years or over $(70$ 's)

\section{INTRODUCTION}

Recently, there are many reports that elective one-stage resection and anastomosis has been performed after bowel decompression using an ileus tube, perianal stent expansion (1-4), or an artificial anus for obstructed colorectal cancer from the fear of failure of the sutures at the time of emergency surgery.

Our hospital is a regional emergency hospital
Abbreviations

CT : computed tomography ; $\mathrm{CF}$ : colon fiberscopy ; SSI : surgical site infection ; IVH : intra venous hyper-alimentation ; D1 : dissection of para-intestinal lymph nodes ; D2 : dissection of para-intestinal and intermediate lymph nodes ; UFT : oral anticancer drug improved from 5 -fluorouracil ; FU : fluorouracil ; LV : leucovorin ; TS-1 : Taihou-Shirasaka-1, novel oral fluoropyrimidine derivative ; FOLFOX : chemotherapy with oxaliplatin plus fluorouracil and leucovolin; QOL : quality of life ; MP : tumor invasion of muscularis propria ; ERAS : enhanced recovery after surgery
Received for publication May 13, 2013 ; accepted June 12, 2013.

Address correspondence and reprint requests to Seiki Tashiro, $\mathrm{MD}, \mathrm{PhD}$, Professor Emeritus, the University of Tokushima, Medical Adviser of Taoka Hospital, 4-2-2 Bandai-cho, Tokushima 770-0941, Japan and Fax : +81-88-655-3077.

The Journal of Medical Investigation Vol. 602013 
and has a psychiatric hospital as related facility. A relatively large percentage of elderly patients and/ or patients with psychological disorders have been introduced on that account.

Therefore, there is also the problem of avoiding the progression of muscle weakness, as result of prolonged immobility and dementia, particularly in our elderly patients. Indwelling ileus tube is not used in obstructive colorectal cancer, the one-stage resection and anastomosis has been performed without subtotal or total colectomy in principle in our hospital.

The emergent one-stage resection and anastomosis procedure for obstructed colorectal cancer was verified about postoperative outcome, by dividing the patients into two groups of Group A under the age of 70 and Group B 70 years old and over.

\section{PATIENTS AND METHODS}

Forty patients underwent surgery for obstructive colorectal cancer in the last 10 years (from April 2002 to March 2011). All of the patients underwent the one-stage emergency resection and anastomosis procedure.

In the case of the right-sided colon cancer, a suction tube was inserted into the oral side of the tumor and the contents were sucked out and decompressed manually before anastomosis.

In the case of the left-sided colon cancer, a larger "Jabara" tube (corrugated tube, bellows) was inserted to the oral side of the tumor and feces was discharged manually through the Jabara tube (see Figure 1). If more hard solid feces were present, gut

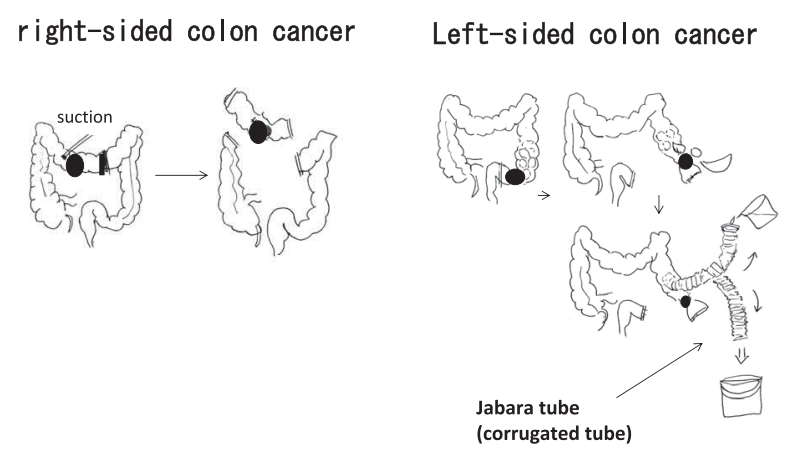

Figure 1. Intraoperative colon decompression method Left illustration shows decompression method with suction tube in right-sided colon cancer, and right illustration shows decompression with Jabara tube in left-sided colon cancer.

There are few patients requiring cleaning with saline and decompression is carried out enough manually. cleaning was performed with saline through the Jabara tube. There were few patients that required cleaning with saline and manual decompression was sufficient.

After decompression in the dilated intestinal tract, ileocolic anastomosis, or colocolnic anastomosis was performed with Albert-Lembert method without using the intestinal tract forceps. First we carried out interrupted suture at the sero-muscular layer of the posterior wall. This was performed by braided 3-0 silk with a control release of a fine needle. Usually 7 8 needles $2-3 \mathrm{~mm}$ in width are finely placed around a $5 \mathrm{~mm}$ portion from the stump, so that dead space is not made in the posterior wall. Next continuous suture of the all layers was performed with 3-0 coated braided absorbable polyglycolic acid at the posterior wall and then in the anterior wall. Finally interrupted suture at the sero-muscular layer of the anterior wall underwent with same way.

When high or low anterior resection underwent, an anastomosis between the rectum and the descending colon was performed using end-to-end anastomosis instrument (EEA).

Postoperative management was performed by enhanced recovery after surgery (ERAS) protocol (5). Namely, a nasogastric tube was not inserted as much as possible, neither was ileus as a result, and was removed immediately after surgery or the next day if needed. Postoperative pain was treated with short-acting anesthetic and epidural analgesia. For shortening of fasting period, fluids are administrated the following day, oral administration is attempted in 3-4 days, early mobilization, and walking.

The characteristics, perioperative parameters and outcome are shown in Table $1,2,3$. The characteristics and surgical outcome between the two groups of younger 70 years old (Group A) and the age of 70 years and older (Group B) were compared using X2 test and Mann-Whitney U-test. Survival curves were calculated using the Kaplan-Meier method and compared using the Wilcoxon test. The statistical significance was defined as $\mathrm{p}<0.05$.

\section{RESULTS}

Forty patients ranged from the ages of 42 years old to 95 years old. The average age was 73.2 years old. Seven of the patients were aged 70-79 years old, 12 of the patients were aged from 80-89 years and 4 patients were 90 years old and over. There were 17 patients in Group A and 23 patients in 
Group B. The elderly percentage of the patients in Group B was 57\%. In addition, there were 7 patients with psychiatric disorders, 6 of 7 patients were aged between $50-60$ years old.

The breakdown of occupied sites are as follows ; left colon cancer tumors were 11 patients from Group A and 13 patients from Group B, and right colon cancer tumors in 6 patients in Group A and 10 in Group B. There was no significant difference between the groups (see Table 1).

Time from hospitalization to surgery ; The shortest time was one hour, and about half of the patients underwent surgery within 6 hours. In total, 34 patients $(85 \%)$ had surgery within 24 hour period.

Diagnosis of obstructive colorectal cancer ; Surgery was performed by necessary minimum examination required, through there were many elderly patients and/or psychiatric disorders. CT was carried out on all patients. 27 patients underwent CF. 1 patient had romanoscopy and 1 had enema. Then $65 \%$ of the patients were diagnosed with only CT scans (Figure 2).

Preoperative albumin level is shown in Table 2. Albumin levels of less than $3 \mathrm{~g} / \mathrm{dl}$ were observed in 7 patients, relatively small $17.7 \%$ and albumin levels of more than $3 \mathrm{~g} / \mathrm{dl}$ were observed in $33 \mathrm{pa}-$ tients $(82.5 \%)$, and 24 patients $(60 \%)$ had albumin level of more than $3.5 \mathrm{~g} / \mathrm{dl}$ (almost normal level). Therfore, nutritional state including albumin was relatively well at admission.

Operation methods are as follow ; Ileocecum resection conducted on 1 patient, Right hemicolectomy on 6 patients, Transverse colon resection on 8 patients, Left hemicolectomy on 5 patients, Sigmoidectomy on 11 patients, High anterior resection in 1 patient, Low anterior resection on 8 patients. And emergent one-stage resection and anastomosis was carried out without covering colostomy or Hartmann's operation in all of the 40 patients.

According to lymph node dissection (6), 10 patients in Group A underwent D1 dissection and 18 patients in Group B, and then 7 patients in Group A and 5 patients in Group B underwent D2 dissection. There were no significant differences between the two groups (Table 1).

According to curability, CurA 11 patients CurB 1 patient CurC 5 patients in Group A, CurA 21 patients CurB 1 patient CurC 1 patient in Group B.

The median operation time was 147.5 minutes in Group A, and 122 minutes in Group B. Operation time in Group B was significantly shorter.

There were no hospital deaths as a result in either
Table 1 Patient's Characteristics

\begin{tabular}{|c|c|c|c|}
\hline & $\begin{array}{l}\text { under } 70 \text { years } \\
\text { old (Group A, } \\
17 \text { patients) }\end{array}$ & $\begin{array}{l}\text { the age of } 70 \text { years } \\
\text { or older (Group B, } \\
23 \text { patients) }\end{array}$ & $\mathrm{p}$ \\
\hline \multicolumn{4}{|l|}{ Lesion } \\
\hline left colon & $11(64.7 \%)$ & $13(56.5 \%)$ & 0.428 \\
\hline right colon & $6(35.3 \%)$ & $10(43.3 \%)$ & 0.6015 \\
\hline \multicolumn{4}{|l|}{ Serum albumin } \\
\hline $3>$ & $2(11.8 \%)$ & $5(21.7 \%)$ & 0.4118 \\
\hline $3 \leqq \sim<3.5$ & $3(17.6 \%)$ & $6(26.0 \%)$ & 0.5274 \\
\hline $3.5 \leqq \sim<4.0$ & $4(23.5 \%)$ & $8(34.8 \%)$ & 0.6314 \\
\hline $4 \leqq$ & $8(47.0 \%)$ & $4(17.4 \%)$ & 0.043 \\
\hline \multicolumn{4}{|l|}{ LN dissection } \\
\hline D1 & $10(58.8 \%)$ & $18(78.3 \%)$ & 0.1848 \\
\hline D2 & 7 (41.2\%) & 5 (21.7\%) & 0.1848 \\
\hline \multicolumn{4}{|l|}{ Stage } \\
\hline I & $0(0 \%)$ & $0(0 \%)$ & \\
\hline II & $6(35.3 \%)$ & $15(65.2 \%)$ & 0.061 \\
\hline III & $5(29.4 \%)$ & $6(26.1 \%)$ & 0.8159 \\
\hline IV & $6(35.3 \%)$ & $2(8.7 \%)$ & 0.0376 \\
\hline $\begin{array}{l}\text { Median operation } \\
\text { time }\end{array}$ & 147.5 minutes & 122 minutes & 0.0267 \\
\hline $\begin{array}{l}\text { Median hospital } \\
\text { duration }\end{array}$ & 28.5 days & 19.5 days & 0.0019 \\
\hline
\end{tabular}

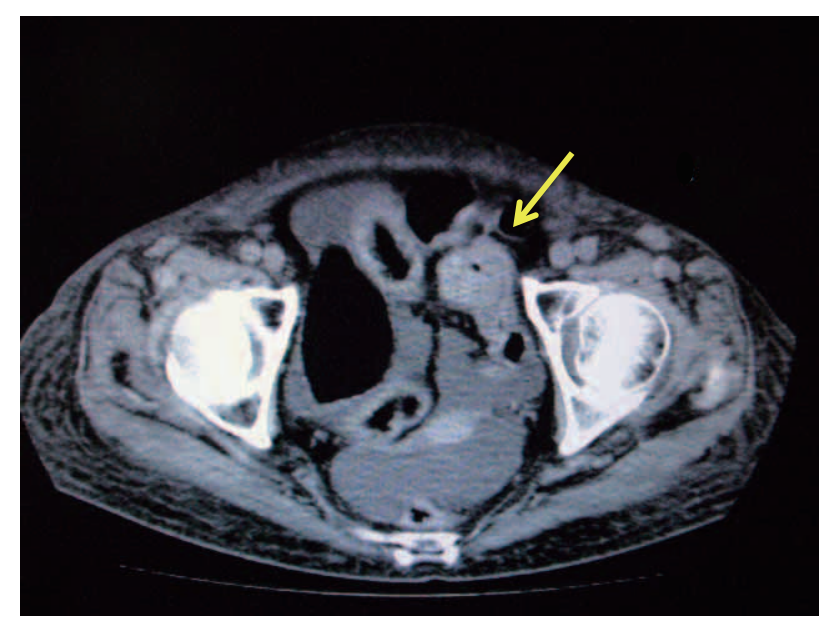

Figure 2. Diagnosis of obstructed colorectal cancer by computed tomography(CT).

Tumor of the sigmoid colon is shown with $\downarrow$ (arrow). Dilatation of the oral side colon is seen, and is not seen in the anal side colon.

Table 2 Preoperative Albumin Level in 40 patients

\begin{tabular}{l|c}
\hline albumin level (g/dl) & patients (\% to 40 all patients) \\
\hline $3>$ & $7(17.5)$ \\
$3 \leqq \sim<3.5$ & $9(22.5)$ \\
$3.5 \leqq \sim<4$ & $12(30.0)$ \\
$4 \leqq$ & $12(30.0)$ \\
\hline
\end{tabular}


of the two groups.

Postoperative complications were observed in 12 of 40 patients (30\%), 4 patients in Group A and in 8 patients in Group B. There were no significant differences between the two groups. Anastomotic leakage was observed in 1 patient from Group A, the patient underwent re-suture on the $6^{\text {th }}$ day (Laparotomy) and was discharged on $15^{\text {th }}$ day after re-surgery. Paralytic ileus was observed in 6 patients (15\%), surgical site infection (SSI) in 1 patient, colitis in 2 patients, pneumonia in 1 patient and liver abscess in 1 patient. All these patients recovered with conservative treatment (Table 3 ).

Table 3 Postoperative Complication

\begin{tabular}{|l|c|c|c|}
\hline Complications & $\begin{array}{l}\text { under 70 years } \\
\text { old (Group A, } \\
\text { 17 patients) }\end{array}$ & $\begin{array}{l}\text { the age of 70 years } \\
\text { or older (Group B, } \\
\text { 23 patients) }\end{array}$ & $\mathrm{p}$ \\
\hline Operation death & 0 & 0 & NS \\
\hline $\begin{array}{l}\text { Anastomotic } \\
\text { leakage }\end{array}$ & 1 & 0 & \\
$\begin{array}{l}\text { Paralytic ileus } \\
\text { Surgical site } \\
\text { infection(SSI) }\end{array}$ & 1 & 5 & \\
$\begin{array}{l}\text { Liver abscess } \\
\text { Pneumonia }\end{array}$ & 1 & 0 & \\
Colitis & 0 & 0 & \\
\hline Total & $4(23.5 \%)$ & $8(34.8 \%)$ & 0.4426 \\
\hline
\end{tabular}

Oen of 5 patients with paralytic ileus and one of 2 patients with colitis were combined with SSI, concomitantly.

The median hospital duration was 28.5 days in Group A and 19.5 days in Group B. The hospitalization period of elderly patients in Group B was significantly shorter.

In regard to histological stage according to Japanese Classification of Colorectal Carcinoma (6), stage II was observed in 6 patients (35.3\%) of Group A and 15 patients (65.2\%) in Group B, stage III in 5 patients (29.4\%) in Group A and 6 patients (26.1\%) in Group B, and stage IV in 6 patients (35.3\%) in Group A and 2 patients (8.7\%) in Group B. Stage II or III did not have any difference between the two groups, but there was quite a significance in Group A for stage IV.

Overall survival rate after 5 years showed $41.8 \%$ in all patients, and 5 year survival rates in stage II were $69.2 \%, 44.0 \%$ in stage III, and $0 \%$ in stage IV (Figure 3).

Overall (whole stage) survival curves in Group A and Group B were not significantly different between the two groups (Figure 4).

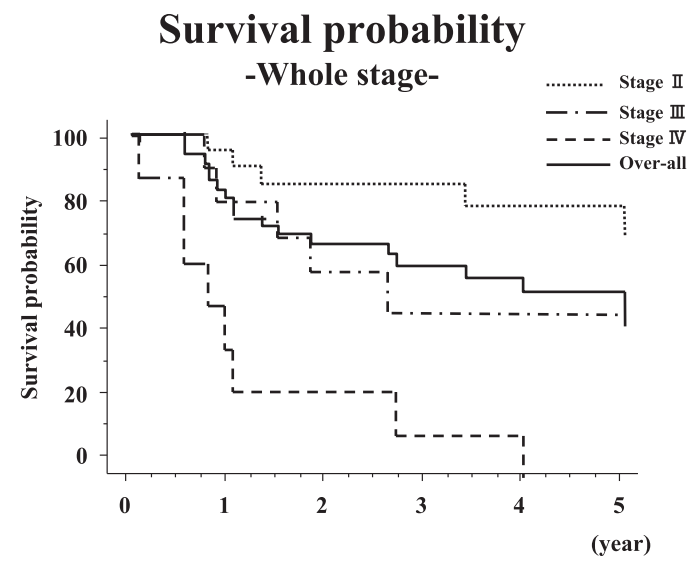

Figure 3. Overall Kaplan-Meier survival curves were showed in the whole stage, stage II, III and IV. Overall 5 year survival rates showed $41.8 \%$, and 5 year survival rates were $69.2 \%$ in stage II, $24.0 \%$ in stage III, and $0 \%$ in stage IV. And the long term survival was bad in stage III and IV.

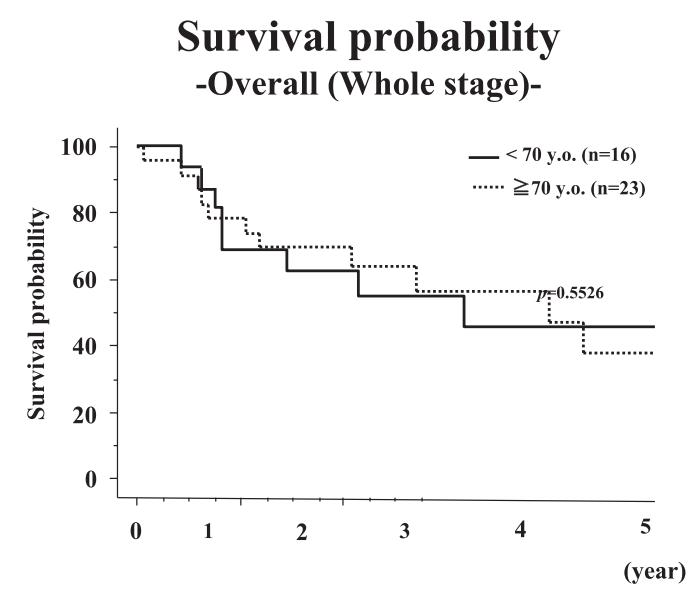

Figure 4. Overall (whole stage) survival curves of under 70 years old (Group A) and the age 70 years or older (Group B) were not significantly different between the two groups.

Stage II or III did not have differences between the two groups (Figure 5,6). There were significantly low in Group B in stage IV (Figure 7), but case is small and one of two cases was not cancerspecific death.

In summary, one-stage resection and primary anastomosis for obstructed colorectal cancer could be applied safely in emergency patients and in turn allowed achieving excellent short-term results in even elderly patients.

It would be valid to state that one stage repair anastomosis after resection shortens the period of fasting, prevents progressive muscle weakness and dementia and avoids prolonged immobility particularly in elderly patients and/or patients with psychiatric disorders. 


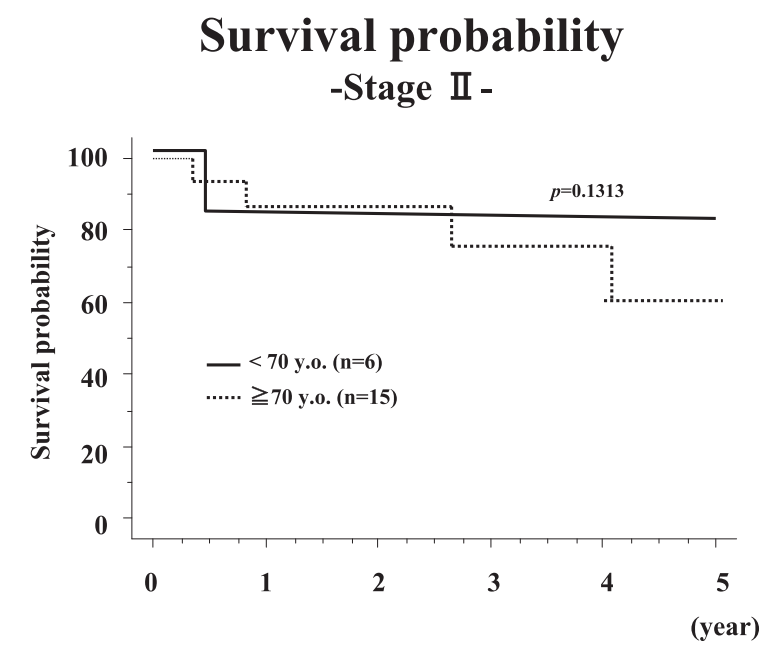

Figure 5. Survival curves of stage II in the two groups showed no significantly difference.

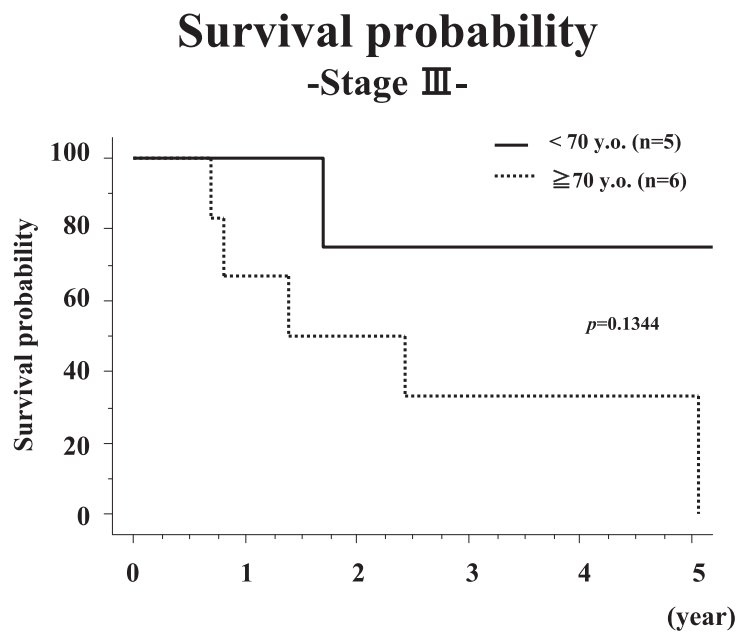

Figure 6. Survival curves of stage III in the two groups showed no significantly difference.

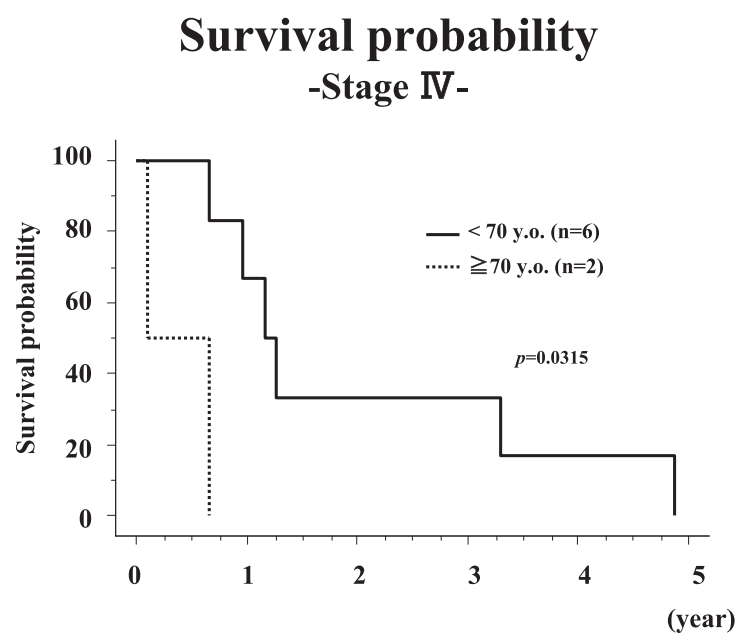

Figure 7. Survival curves of stage IV in group B ( $70 \leqq$ years old) showed significantly lower than Group A (70> years old).

\section{DISCUSSION}

Because of the fear of the suture failure at the time of the emergent one-stage resection and anastomosis, recently elective one-stage resection and anastomosis has increased after intestinal decompression by the construction of artificial anus (7-11), and trans-anal tube and stent $(1-4,12)$. However, until the operation the QOL of the patients was affected because of the difficulty in managing the daily frequency of stools.

Albumin levels of less than $3 \mathrm{~g} / \mathrm{dl}$ were observed in only 7 patients (17.7\%), and 24 patients $(60 \%)$ had albumin levels of more than $3.5 \mathrm{~g} / \mathrm{dl}$ (almost normal level) at admission to our hospital.

Sumi et al. (13) reported that a preoperative albumin level before the operation fell compared with the time of hospitalization in $62.5 \%$ of patients, in spite of having performed enteral nourishment and/ or IVH during preoperative intestinal decompression by trans-anal drainage tube before colonic resection. Therefore, surgery should be performed as soon as possible to break the ileus. There is growing acceptance regarding one-stage resection and primary anastomosis for the majority patients with obstructed colorectal cancer in some reports (14-18). These reports suggest that primary anastomosis can be performed safely with minimum morbidity and mortality.

Intestinal decompression is required for emergency one-stage anastomosis before resection. There are manual decompression and colonic irrigation with on-table lavage as a method. Manual decompression was comparable to colonic irrigation of primary anastomosis in obstructed colorectal emergency, with no significant increase mortality, leak or infection rates in the literatures (18-22). We enforced a manual decompression on all patients and by doing this we were able to achieve very good results.

The disadvantage of this procedure is that we can't verify by preoperative examination whether there are synchronous cancers in the proximal colon that obstruct or not. During the manual decompression, palpation should be done carefully, because invasive cancer over muscularis propria (MP, indication of operative resection) is diagnosed by palpation. Endoscopic examination is done after surgery, and if small enough, endoscopic removal is possible.

One-stage resection and primary anastomosis had excellent short-term results. A mortality rate at least 
two to three times higher among the elderly (70 and over) than in younger patients has been reported repeatedly in various populations (23). In our series, there were many elderly patients aged 70 and over (about 60\%). Operative mortality was still 0\%. The main reason for this is the gentle manual decompression and polite operation, and introduction of enhanced recovery after surgery (ERAS) protocol (5).

However, emergency surgery for obstructed colorectal cancer was associated with an inferior longterm survival ratio especially in stage III, IV of the series. There are some reports (24-26) that the short-term was good, but the long-term rate was worse. In addition there is a report that in retrospective comparison between bridge surgery after selfexpanding metallic stent (SEMS) group and surgery only group, overall survival $(p=0.001)$ and a 5 -year overall survival $(p=0.0003)$ were significantly lower in the SEMS group than in the surgery-only group, and a 5 -year cancer-specific mortality was significantly higher in the SEMS group ( $48 \%$ vs $21 \%$, respectively $(\mathrm{p}=0.02)(27)$.

We also have many elderly peoples, some patients might have cancer-nonrelated death. However, $70 \%$ of patients underwent D1 lymph nodes dissection. Lykke J et al. (28-30) reported that high lymph node count harvested (12 more over) was associated with improved overall survival in colon cancer. Therefore the lymph node dissection over D2 and R0 curative resection should be done to prevent loco-regional recurrence.

Adjuvant chemotherapy was carried out postoperatively for 6 months in the patients with more than stage III in principle, taking into account the risk of cases. Actually as adjuvant chemotherapy in stage III, UFT or 5-FU+LV was enforced in 2 patients, TS- 1 was administrated in 4 patients, FOLFOX6 was enforced in 1 patient and was not given to 4 patients. In stage IV, FOLFOX6 was given to 7 patients, and was not given to 1 patient. Systemic chemotherapy was not performed under these circumstances in our clinic. Therefore, postoperative adjuvant chemotherapy should be formed systemically in patients with more stage III and stage II with high risk of progression.

\section{CONFLICT OF INTEREST STATEMENT}

Yuko Sumise and other co-authors have no conflict of interest.

\section{REFERENCES}

1. Feo L, Schaffzin DM : Colonic stent : the modern treatment of colonic obstruction. Adv Ther 28(2) : 73-86, 2011

2. Dulucq J L, Wintringer P, Beyssac R, Barberis C, Talbi P, Mahajna A : One-stage laparoscopic resection after placement of self-expanding metallic stent for colorectal obstruction. Dig Dis Sci $51: 2365-71,2006$

3. Lee WS, Beak JH, Kang JM, Kwon KA: The outcome after stent placement or surgery as the initial treatment for obstructive primary tumor in patients with stage IV colon cancer. Am J Surg 203 : 715-19, 2012

4. Zhang Y, Shi J, Shi B, Song CY, Xie WF, Chen YX : Self-expanding metallic stent as a bridge to surgery versus emergency surgery for obstructive colorectal cancer: a metaanalysis. Surg Endosc 26 : 110-9, 2012

5. Fearon K C , Ljungvist O, Von Meyenfieldt M, Revhaug A, Dejong CH, Lassen K, Nygren J, hausel J, Soop M, Anderson J, Kehlet H : Enhanced recovery after surgery : A consensus review of clinical care for patients undergoing colonic resection. Clin Nutr 24 : 466-77, 2005

6. Japanese Society for Cancer of the Colon and Rectum (ed.) (2009) General Rules for Clinical and Pathological on Cancer of the Colon, Rectum and Anus. The $7^{\text {th }}$ Edition, Revised Version. Kanehara, Tokyo

7. Guennags KF, Lustosa SA, Saad SS, Saconato $\mathrm{H}$, Matos D : Ileostomy or colostomy for temporary decompression of colorectal anastomosis. Acta Cir Bras 23 : 294-303, 2008

8. Hennekkinne-Mucci S, Tuech J J, Brehant O, Lemite E, Bergamaschi R, Pessaux P, Arnaud JP : Emergency subtotal/total colectomy in the management of obstructed left colon carcinoma. Int J Colorectal Dis 21 : 538-41, 2006

9. Balogh A, Wittmann T, Varga L, Zollei I, Lazar G, Baradnay G, Rosztoczy A, Molnar T, Tiszlavicz L, Kiss Zsuzsa F, Nagy F : Subtotal colectomy for the treatment of obstructive left colon cancer. Follow-up results. Ory Hetil 143 : 1577-83, 2002

10. Hennekine-Mucci S, Tuech J J, Brehant O, Lermite E, Pessaux P, Lada P, Hamy A, Arnaud JP : Management of obstructed left colon carcinoma. Hepatogastroenterology 54 : 1098-101, 2007

11. Vukovic M, Molievic $\mathrm{N}$ : Total colectomy-option 
in management of acute obstruction of the leftside colon. Med Preol 6 : 43-7, 2008

12. Fischer A, Schrag HJ, Goos M, Obermaier R, Hopt UT, Baier PK : Transanal endoscopic tube decompression of acute colonic obstruction : experience with 51 cases. Surg Endosc 22 : 683-8, 2008

13. Sumi K, Yamaji K, Mukae Y, Yakabe T, Koga $\mathrm{Y}$, Noshiro H : management of acute obstruction caused by left-sided colorectal carcinoma (in Japanese with English abstract). J Abdom Emerg Med 30 : 765-71, 2010

14. Hsu T C : Comparison of one-stage resection and anastomosis of acute complete obstruction of left and right colon. Am J Surg 189 : 384-7, 2005

15. Gatsoulis N, Roukounakis N, Kafetzis I, Mavrakis G : Surgical management of large bowel obstruction due to colonic cancer. Tech Coloproctol $1: \mathrm{s} 82-4,2004$

16. Iancu C, Osian G, Mocan L, Mocan T, Zaharie F, Todea-Iancu D, Bala O, Bodea R, Al-Hajjar N, Pop F, Puia IC, Graur F, Munteanu D, Vlad $\mathrm{L}$ : Management of colorectal resections for treatment of neoplastic intestinal occlusion. Experience of surgery clinic No III, Cluj-Napoca. Chirurogia(Bucur) $103:$ 45-51, 2008

17. Naraynsingh V, Rampaut R, Maharaj D, Kuruvilla T, Ramcharan K, Pouchet B : prospective study of primary anastomosis without colonic lavage for patients with an obstructed left colon. Br J Surg 86 : 2341-3, 1999

18. Cross KL, Rees JR, Soulsby RH, Dixon AR: Primary anastomosis without colonic lavage for obstructed left colon. Ann R Coll Surg Engl 90 : 302-4, 2008

19. Lim JF, Tanq CL, Seow-Choen F, Heah SM : Prospective, randomized trial comparing intraoperative colonic irrigation with manual decompression only for obstructed left left-sided colorectal cancer. Dis Colon Rectum 48 : 205-9, 2005

20. Ansaloni L, Andersson RE, Bazzoli F, Catena F, Cennamo V, Di Saverio S, Fuccio L, Jeekel H, Leppaniemi A, Moore E, Pinna AD, Pisano M, Repici A, Sugarbaker PH, Tuech JJ : Guidolenines in the management of obstructing cancer of the left colon : consensus conference of the world society of emergency surgery (WSES) and peritoneum and surgery (PnS) Society. World J Emerg Surg 28 : 5-29, 2010

21. Sule AZ, Missauno M, Opaluwa AS, Ojo E: One-stage treatment of left-sided large bowel emergencies. East Afr med J 85 : 90-4, 2009

22. Kam MH, Tang CL, Chan E, Lim JF, Eu KW : Systematic review of intraoperative colonic irrigation vs. manual decompression in obstructed left-sided colorectal emergencies. Int J Colorectal Dis $24: 1031-7,2009$

23. Iversen LH : Aspects of survival from colorectal cancer in Denmark. Dan Med J 59 : B4428, 2012

24. Baccari P, Bisagni P, Crippa R, Sampietro R, Staudacher $\mathrm{C}$ : Operative and long0term results after one-stage surgery for obstructing colonic cancer. Hepatogastroenterology 53 : 698-701, 2006

25. Runkel N S, Schlag P, Schwarz V, Herfarth C : Outcome after emergency surgery for cancer of the large intestine. Br J Surg $78: 183-8,1991$

26. Ohman $\mathrm{U}$ : Prognosis in patients with obstructing colorectal carcinoma. Am J Surg 143 : 7427,1982

27. Sabbagh C, Browel F, Diouf M, Cosse C, Brehant O, Bartoli E, Mauvais F, Chauffert B, Dupas JL, Nguyen-Khac E, Regimbeau JM : Is stenting as "a bridge to surgery" an oncologically safe strategy for the management of acute, left-sided, Malignant, colonic obstruction? : A comparative study with a propensity score analysis. Ann Surg Jan 15., 2015 (Epub ahead of print)

28. Lykke J, Roikjaer O, Jess $\mathrm{P}$ : The relation between lymph node status and survival in stage i-iii coloncancer : results from a prospective natinalwide cohort study. Colorectal Dis Oct 12 , 2012

29. Sjo OH, Merok MA, Svindland A, Nesbakken A : Prognostic impact of lymph node havest and lymph node ratio in patients with colon cancer. Dis Colon Rectum 55 : 307-15, 2012

30. Wong SL: Lymph node counts and surgical rates after resection for colon and rectal cancer. J Natl cancer inst 99 : 433-41, 2007 